\title{
Effet des Doses de Semis et de la Méthode De Conduite de La Pépinière sur les Caractéristiques Morphologiques des Plantules de Cinq Variétés D’oignon (Allium cepa L.) en Côte d'Ivoire
}

\author{
Koffi Eric-Blanchard Zadjéhi, \\ Diarrassouba Nafan, \\ Yao Saraka Didier Martial
}

Université Peleforo Gon Coulibaly (UPGC), UFR Sciences Biologiques, Département de Biochimie-Génétique, Unité Pédagogique et de Recherche

(UPR) de Génétique, Korhogo, Côte d’Ivoire

Sie Raoul Sylvère

Université Nangui-Abrogoua, Abidjan, Côte d’Ivoire

Doi:10.19044/esj.2021.v17n34p208

Submitted: 05 May 2021

Accepted: 15 September 2021

Published: 30 September 2021
Copyright 2021 Author(s)

Under Creative Commons BY-NC-ND 4.0 OPEN ACCESS

Cite As:

Koffi E-B.Z., Diarrassouba N., Yao S.D.M. \& Sie R.S. (2021). Effet des Doses de Semis et de la Méthode De Conduite de La Pépinière sur les Caractéristiques Morphologiques des Plantules de Cinq Variétés D’oignon (Allium cepa L.) en Côte d'Ivoire. European Scientific Journal, ESJ, 17(34), 208.

https://doi.org/10.19044/esj.2021.v17n34p208

\section{Résumé}

Le manque de méthodes standardisées pour la pépinière d'oignon a suscité la présente étude. Elle a pour objectif d'évaluer deux pratiques culturales de pépinière d'oignon à travers les caractéristiques végétatives des plantules. À cet effet cinq variétés (Bati, Damani, Cara, Karibou et Safari) et deux milieux de conduite de pépinière : terre et alvéole ont été mis en comparaison sur le site expérimental de l'Université Peleforo GON COULIBALY de Korhogo de janvier à février 2020. Sur terre, trois doses de semis $(3,4$ et $5 \mathrm{~g} / \mathrm{m} 2)$ et dans les alvéoles trois graines par poquet ont été expérimentées. Les données morphologiques ont été collectées 40 jours après semis. Le logiciel SPSS 22.0 a été utilisé pour le traitement statistique des données collectées. Une analyse de variance incorporant la comparaison des moyennes selon Newman et Keuls au seuil de 5\% a été réalisée pour comparer les caractéristiques morphologiques des différentes variétés entre elles. Il 
ressort de cette étude que les plantules élevées dans les alvéoles ont eu en moyenne les tailles les plus élevées $(17,32 \mathrm{~cm})$. Sur terre, les tailles ont varié de 14,77 cm (dose de $05 \mathrm{~g} / \mathrm{m} 2$ ) à 16,09 cm (dose 03 et $04 \mathrm{~g} / \mathrm{m} 2$ ). Les doses de semis 03 et $04 \mathrm{~g} / \mathrm{m} 2$ ont enregistré les plantules les plus robustes relativement au nombre de feuilles (03), les poids frais $(0,35 \mathrm{~g})$ et sec $(0,035 \mathrm{~g})$ et le taux de matière sèche (10\%). Les variétés Damani, Karibou et Safari ont eu les meilleures performances relativement aux caractéristiques morphologiques. La robustesse de la plantule est corrélée positivement à sa taille et au nombre de feuilles. Au vu des résultats, la pépinière d'oignon peut être conduite dans les alvéoles ou sur terre à la dose $04 \mathrm{~g} / \mathrm{m} 2$. La sélection des plantules en pépinière peut se faire en se basant uniquement sur la taille de la plantule et le nombre de feuilles.

Mots clés: Oignon, Méthode Pépinière, Caractères Végétatifs, Dose De Semis, Sélection De Variété

\title{
Effect of Sowing Doses and Nursery Management Method on Morphological Characteristics of Seedlings of Five Onion (Allium cepa L.) Varieties in Côte d'Ivoire
}

\author{
Koffi Eric-Blanchard Zadjéhi, \\ Diarrassouba Nafan, \\ Yao Saraka Didier Martial
}

Université Peleforo Gon Coulibaly (UPGC), UFR Sciences Biologiques, Département de Biochimie-Génétique, Unité Pédagogique et de Recherche (UPR) de Génétique, Korhogo, Côte d’Ivoire

Sie Raoul Sylvère

Université Nangui-Abrogoua, Abidjan , Côte d'Ivoire

\begin{abstract}
The lack of standardized methods for the onion nursery prompted this study. Its objective was to evaluate two cultivation practices of the onion nursery through the vegetative characteristics of the seedlings. For this purpose, five varieties (Bati, Damani, Cara, Karibou and Safari) and two nursery management: soil and well plate, were compared on the experimental site of Peleforo GON COULIBALY University in Korhogo from January to February 2020. On soil, three doses of seeding (3, 4 and $5 \mathrm{~g} / \mathrm{m} 2)$ and, in the plate, 3 seeds/holes were tested. Morphological data were collected 40 days after sowing. In this sample, SPSS 22.0 software was used for statistical data processing. An analysis of variance incorporating the comparison of means according to Newman and Keuls at the 5\% threshold was used to compare the
\end{abstract}


morphological characteristics of the different varieties. It appears from this study that the seedlings reared in the well plate were averagely the highest with $17.32 \mathrm{~cm}$. On soil, the heights varied from $14.77 \mathrm{~cm}$ (dose $05 \mathrm{~g} / \mathrm{m} 2$ ) to $16.09 \mathrm{~cm}$ (dose 03 and $04 \mathrm{~g} / \mathrm{m} 2$ ). Seeding doses of 03 and $04 \mathrm{~g} / \mathrm{m} 2$ recorded the most robust seedlings in relation to the number of leaves (03), fresh $(0.35$ g) and dry (0.035 g) weights and dry matter rate of $10 \%$. The Damani, Karibou and Safari varieties had the best performances concerning morphological characteristics. The robustness of the seedling is positively related to its size and the number of leaves. Besides well pate, a seeding rate of $04 \mathrm{~g} / \mathrm{m} 2 \mathrm{can}$ be recommended for the management of the nursery on soil. The selection of seedlings in the nursery can be done based only on the size of the seedling and the number of leaves.

Keywords: Onion, Nursery Method, Vegetative Characteristics, Sowing Rate, Selection Of Variety

\section{Introduction}

L'oignon occupe une place très importante dans l'agriculture mondiale. Il est cultivé dans le monde entier notamment dans les régions chaudes. Cette culture présente de bonnes perspectives économiques, vu la superficie cultivée en pleine croissance avec des productions étalées sur une longue période de l'année (David-Benz \& Seck 2018 ; Laouali et al., 2019). En Afrique, la culture de l'oignon contribue significativement à l'autonomisation du monde rural et surtout de la femme (Kiema, 2019).

En Côte d'Ivoire, la consommation de l'oignon s'est développée depuis longtemps sur la base de l'importation. Sur les 50 dernières années, les importations d'oignon connaissent une croissance quasi exponentielle avec une nette accélération depuis le début des années 2000. En 2013, l’oignon était le troisième produit alimentaire le plus importé par la Côte d'Ivoire après le riz et le blé (RONGEAD, 2014).

En 2014 avec une production estimée entre 5000 et 7500 tonnes, la Côte d'Ivoire reste l'un des pays petits producteurs de la sous-région malgré un potentiel de production important aussi bien en zones de savanes qu'en zones forestières (RONGEAD, 2014). La production de semences et de plans maraîchers est une activité d'une importance économique tout à fait particulière. Le rendement et la qualité de la production d'oignon sont étroitement liés à la qualité de la semence et à la période de production. Des expérimentations ont été faites dans plusieurs pays en vue d'optimiser le rendement et ce, en se focalisant sur le choix variétal, l'itinéraire technique et les travaux culturaux (Silué et al., 2003, Bello et al., 2019a). En somme, le rendement d'un champ d'oignon dépend en grande partie du choix des variétés adaptées à la zone de culture et surtout de l'itinéraire technique adopté. La 
conduite de la pépinière occupe une place non négligeable dans l'itinéraire technique. En effet, la réussite du champ dépend en grande partie de la qualité des plants élevés en pépinière. À ce jour en Côte d'Ivoire, aucune méthode validée de conduite de pépinière en vue de proposer le meilleur rendement (en quantité et en qualité) n'est disponible dans le monde agricole pour la culture de l'oignon. C'est pour remédier à ce manque d'information que cette expérimentation a été conduite. Elle vise à évaluer deux méthodes de conduite de pépinière et à faire des propositions relativement à la quantité de graines.

\section{Matériel et méthodes}

\section{Site d'expérimentation}

L'essai a été conduit sur la station expérimentale de l'Université Peloforo GON COULIBALY (UPGC) de Korhogo dans le Nord de la Cote d'Ivoire (Figure 1). La pluviosité annuelle varie entre 1200 et $1400 \mathrm{~mm}$. Le climat est de type tropical soudano-guinéen, marqué par deux grandes saisons, une pluvieuse qui s'étend de mai à octobre et une sèche de novembre à avril. Le sol du site expérimental est de type rouge sablo argileux avec environ 88\% de sable (Siéné et al., 2020).

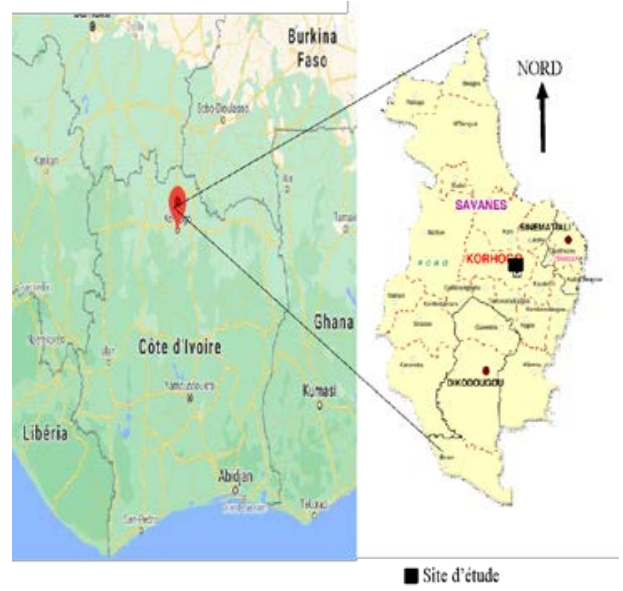

Figure 1. Localité de Korhogo et site d'étude en Côte d'Ivoire Source: Image projet de recherche sur l'oignon de l’UPGC (2020)

\section{Matériel végétal}

Le matériel végétal utilisé est constitué de cinq variétés d'oignon achetées sur le marché de la ville de Korhogo. Il s’agit des variétés Bati, Karibou, Cara, Damani (type Galmi) et Safari. 


\section{Méthodes}

\section{Dispositif expérimental et conduite de la pépinière sur terre}

Les planches ont une dimension de $100 \mathrm{~cm}$ x $100 \mathrm{~cm} \times 15 \mathrm{~cm}$ (Longueur $\mathrm{x}$ largeur $\mathrm{x}$ hauteur), soit un mètre carré sur une hauteur de $15 \mathrm{~cm}$. Les planches confectionnées ont été binées et nivelées. Une fumure minérale de fond, NPK 15-15-15 a été appliquée à raison de $30 \mathrm{~g} / \mathrm{m}^{2}$, trois jours avant semis. Après apport d'engrais, les planches ont été arrosées.

Le semis a été réalisé dans le mois de janvier 2020 selon un dispositif en trois (3) blocs. Ce dispositif a été choisi pour faciliter la mise en place de l'essai, les calculs statistiques et ce, en raison de sa simplicité et sa possibilité d'application à toutes sorte d'expériences. Il a l'avantage d'être simple à réaliser et permet le contrôle de l'hétérogénéité du sol ainsi que la comparaison entre traitement.

Trois doses de semis (ou quantités de semences) ont été mises en expérimentation. Il s'agit de doses 3,4 et $5 \mathrm{~g} / \mathrm{m}^{2}$. Chaque dose de semence a été répétée 3 fois pour chaque variété. Un total de 45 planches a été donc confectionné pour les cinq variétés. Le dispositif expérimental est présenté sur la figure 2.

Avant le semis, les planches ont été légèrement arrosées pour faciliter le traçage des sillons de semis à l'aide d'un doigt ou un bâton. Sur chaque planche 10 sillons de semis espacés d'environ $10 \mathrm{~cm}$ ont été réalisés. Les graines d'oignons ont été déposées dans les sillons en les séparant le plus possible avant le recouvrement léger de terre (Figure 3). Les planches ont été ensuite paillées. La paille maintient l'humidité pour faciliter la germination. Après germination, des ombrières d'une hauteur d'un (01) mètre ont été confectionnées sur les planches et enlevée après 15 jours.

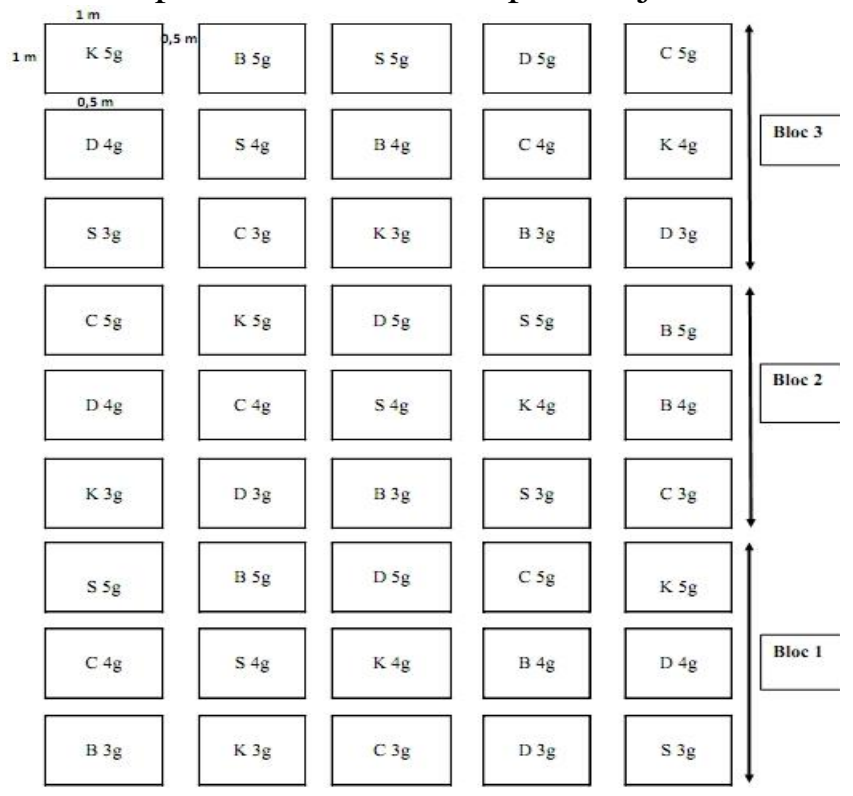


NB : $\mathbf{B}=$ oignon Bati, $\mathbf{K}=$ oignon rouge $\mathbf{K a r i b o u}, \mathbf{C}=$ oignon $\mathbf{C a r a}, \mathbf{D}=$ oignon violet Damani (type Galmi), S=oignon Safari

Figure 2. Dispositif expérimental de la pépinière d'oignon sur terre Source: Figure projet de recherche sur l'oignon de l'UPGC (2020)

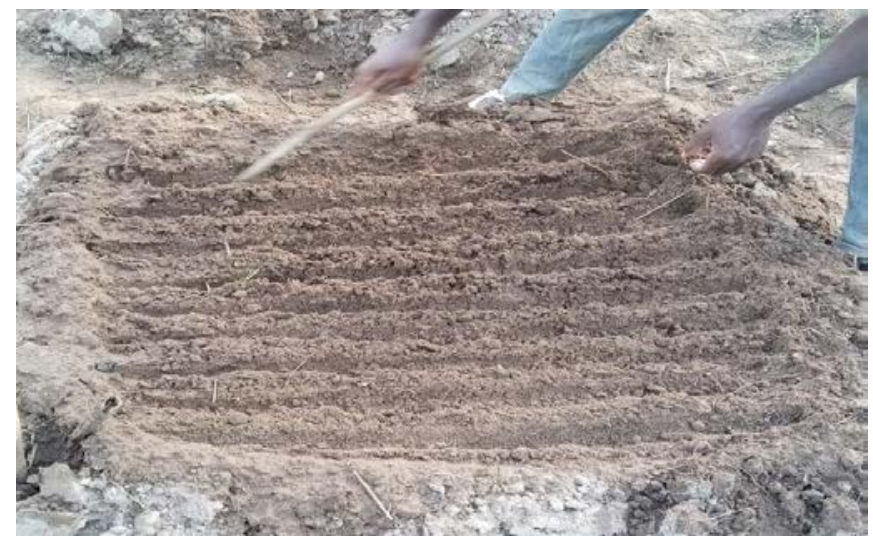

Figure 3. Réalisation de sillons sur les planches pour le semis des graines d'oignon en pépinière sur terre

Source: Photo projet de recherche sur l'oignon de l’UPGC (2020)

\section{Conduite de la pépinière dans des plaques de semis alvéolées}

La pépinière a été conduite dans des plaques industrielles avec 72 alvéoles (Figure 4). Les alvéoles ont été remplis de compost industriel acheté sur le marché de la ville de Korhogo. Le semis a été réalisé à raison de trois graines par alvéole. Les substrats ont été arrosés avant le semis. Un arrosage matin et soir a été réalisé à l'aide d’un pulvérisateur. La pépinière a été conduite sous ombrière.



Figure 4. Alvéoles remplis de substrat pour la conduite de la pépinière

Source: Photo projet de recherche sur l'oignon de l’UPGC (2020)

\section{Collecte des données}

Les caractéristiques morphologiques des plantules ont été mesurées sur des plantules âgées de 40 jours (Figure 5). Dix plantules ont été prélevées par 
planche soit un total de 450 individus pour les 45 planches et 150 individus par dose de semis. Au niveau des plaques de semis, 10 plantules ont été prélevées par variété soit un total de 50 plantules pour les cinq variétés d'oignon. Sur ces plantules, la hauteur a été mesurée à l'aide d'un mètre ruban et le diamètre au collet à l'aide d'un pied à coulisse. Le nombre de feuilles a été compté. Au laboratoire, le poids frais a été déterminé. Les plantules ont été ensuite séchées à l'étuve à $70^{\circ} \mathrm{C}$ pendant 40 heures après avoir été étiquetées. Le poids sec a été ensuite mesuré et le taux de matière sèche a été déterminé en faisant le rapport poids sec sur poids frais.

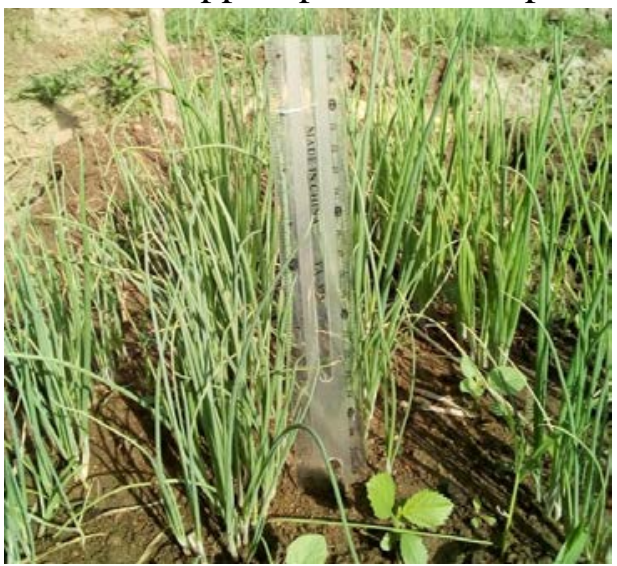

A. Plantules d'oignon sur terre



B. Plantules d'oignon en alvéole

Figure 5. Plantules d'oignon âgées de 40 jours sur terre et en alvéole

Source : Photo projet de recherche sur l'oignon de l'UPGC (2020)

\section{Traitement statistique}

Le logiciel SPSS 22.0 a été utilisé pour les traitements statistiques des données collectées. Les moyennes et les écart-types ont été calculés pour chaque caractère mesuré par dose de semis et par variété. Une analyse de variance incorporant la comparaison des moyennes selon Newman et Keuls au seuil de $5 \%$ a été réalisée pour comparer les caractéristiques morphologiques des différentes variétés entre elles. Le lien entre les variables mesurées a été apprécié à travers le coefficient de corrélation de Pearson (r).

\section{Résultats et discussion}

\section{Résultats}

\section{Effet des doses et le milieu de semis sur les caractères morphologiques des} plantules

Les figures 6 et 7 présentent respectivement, les effets des doses de semis sur la hauteur, le diamètre et le nombre de feuilles de la plantule. Les plantules en alvéole ont eu les hauteurs les plus élevées avec une valeur moyenne de 17,32 $\mathrm{cm}$. Sur terre les plantules de petites tailles ont été observées avec la dose de $05 \mathrm{~g} / \mathrm{m}^{2}$, elles ont une taille moyenne de $14,77 \mathrm{~cm}$. 
La dose $04 \mathrm{~g} / \mathrm{m}^{2}$ a enregistré la valeur la plus élevée pour la hauteur de la plantule sur terre $(16,09 \mathrm{~cm})$. Cette valeur n'est pas significativement différente de celle de la dose $03 \mathrm{~g} / \mathrm{m}^{2}$. La dose $04 \mathrm{~g} / \mathrm{m}^{2}$ a eu également les plantules les plus vigoureuses avec des valeurs élevées de diamètres au collet $(0,21 \mathrm{~cm})$. Pour ce caractère les plus faibles valeurs ont été observées avec la dose de semis $05 \mathrm{~g} / \mathrm{m}^{2}$ avec $0,18 \mathrm{~cm}$. La dose $03 \mathrm{~g} / \mathrm{m}^{2}$ et les plantules en alvéole ont eu les mêmes performances avec des valeurs comprises entre celles des doses 04 et $05 \mathrm{~g} / \mathrm{m}^{2}$. Toujours sur terre, les plantules de la dose $03 \mathrm{~g} / \mathrm{m}^{2}$ ont produit plus de feuilles que celles des autres dosages. Ces plantules ont produit environ 03 feuilles. Les plantules des alvéoles ont enregistré les plus faibles valeurs pour ce caractère soit en moyenne 02 feuilles.

Les figures 8 et 9 présentent respectivement, les effets des doses de semis et le milieu de culture sur les poids frais, sec et le taux de matière sèche de la plantule. Les poids frais et sec puis le taux de matière sèche des plantules d'oignon ont été les plus élevés pour les doses 03 et $04 \mathrm{~g} / \mathrm{m}^{2}$. Les plantules de ces deux doses de semis ont eu un poids frais moyen de 0,35 g, un poids sec de $0,034 \mathrm{~g}$ et un taux de matière sèche d'environ $10 \%$. À l'opposé, les plantules des alvéoles et celles de la dose de $05 \mathrm{~g} / \mathrm{m}^{2}$ ont eu les plus faibles valeurs. Elles ont enregistré un poids frais de $0,30 \mathrm{~g}$, un poids sec de $0,025 \mathrm{~g}$ avec un taux de matière sèche d'environ $09 \%$.
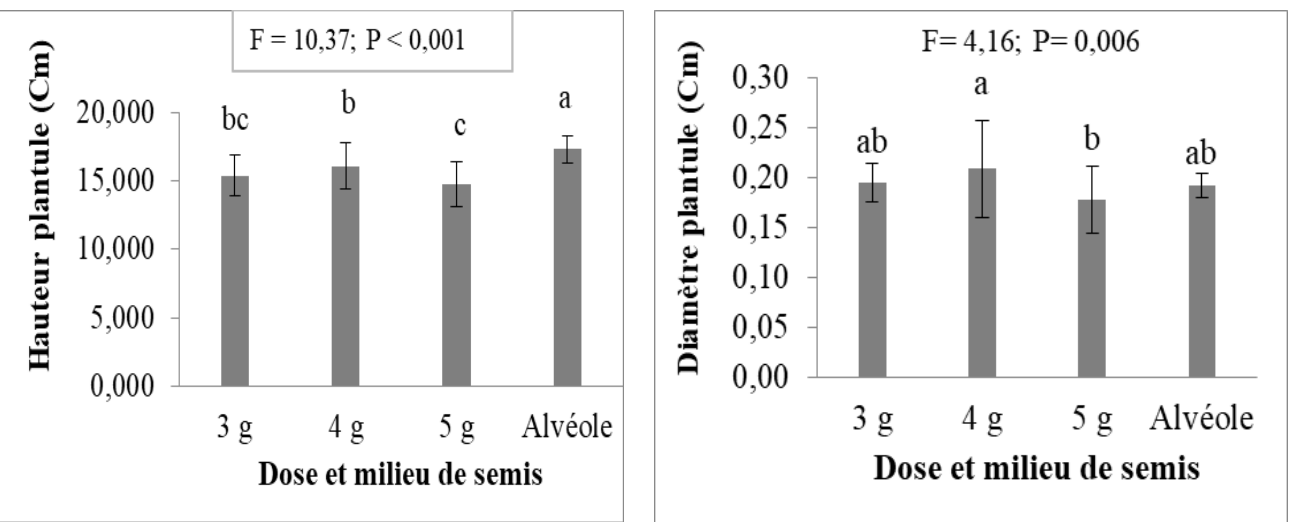

Figure 6. Effet des doses de semis (sur terre et en alvéole) sur la hauteur et le diamètre de la plantule d'oignon en pépinière

NB : Les histogrammes affectés de la même lettre sont statistiquement égaux au seuil $\alpha=5$ $\%$ selon le test de Newman et Keuls. $\mathrm{F}=$ Statistique du test ; $\mathrm{P}=$ probabilité.

Source : Résultat projet de recherche sur l'oignon de l’UPGC (2020) 


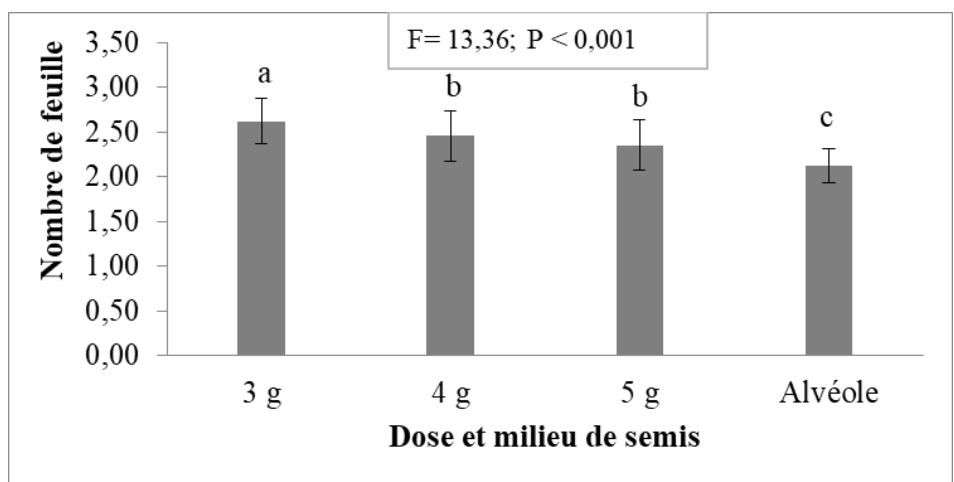

Figure 7. Effet des doses de semis sur terre et en alvéole sur le nombre de feuilles de la plantule d'oignon en pépinière

NB : Les histogrammes affectés de la même lettre sont statistiquement égaux au seuil $\alpha=5$ \% selon le test de Newman et Keuls. $\mathrm{F}=$ Statistique du test ; $\mathrm{P}=$ probabilité.

Source: Résultat projet de recherche sur l’oignon de l’UPGC (2020)
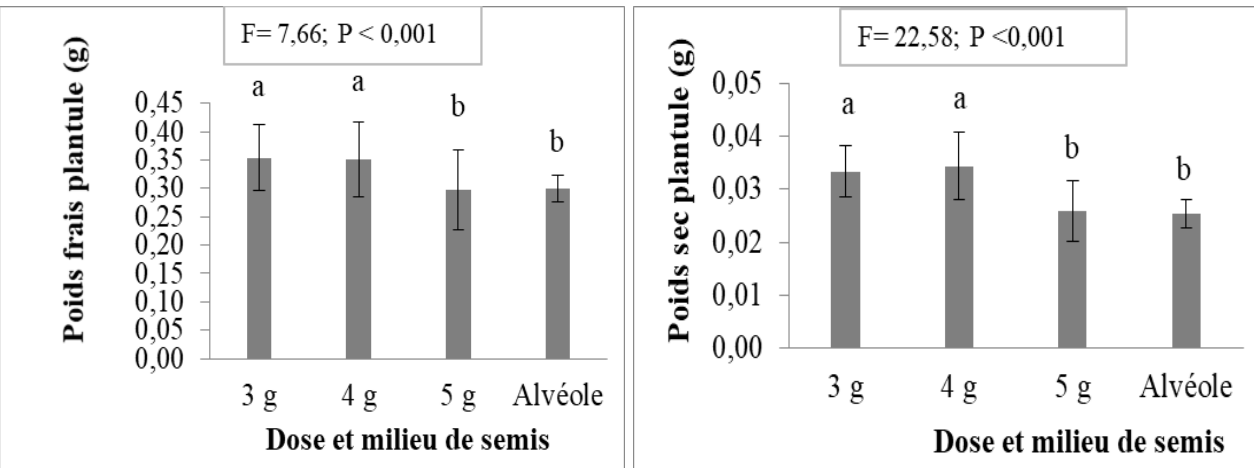

Figure 8. Effet des doses de semis sur terre et en alvéole sur les poids frais et sec de la plantule d'oignon en pépinière

NB : Les histogrammes affectés de la même lettre sont statistiquement égaux au seuil $\alpha=5$ \% selon le test de Newman et Keuls. $\mathrm{F}=$ Statistique du test ; $\mathrm{P}=$ probabilité.

Source: Résultat projet de recherche sur l'oignon de l’UPGC (2020)

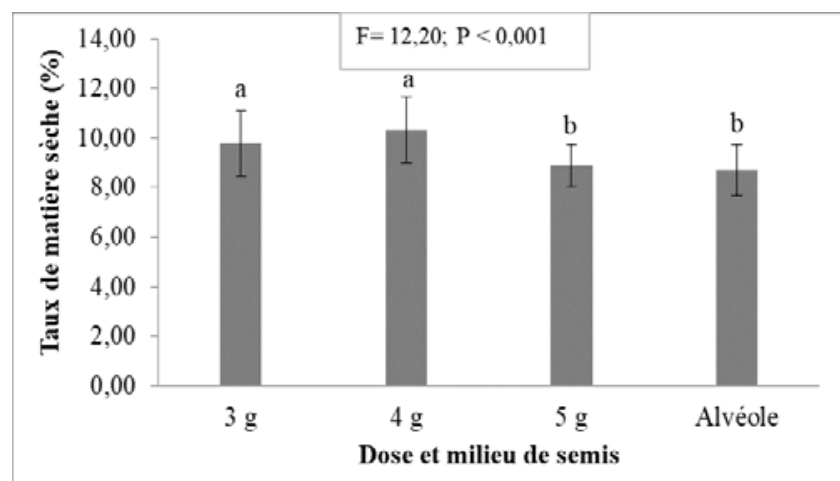

Figure 9. Effet des doses de semis sur terre et en alvéole sur le taux de matière sèche de la plantule d'oignon en pépinière 
NB : Les histogrammes affectés de la même lettre sont statistiquement égaux au seuil $\alpha=5$ \% selon le test de Newman et Keuls. $\mathrm{F}=$ Statistique du test ; $\mathrm{P}=$ probabilité.

Source: Résultat projet de recherche sur l'oignon de l’UPGC (2020)

\section{Comparaison des caractères morphologiques des cinq variétés d'oignon en pépinière}

Les tableaux 1 et 2 présentent les caractéristiques morphologiques des cinq variétés d'oignon en pépinière sur terre et en alvéole. Les variétés Damani, Karibou et Safari semées directement sur le sol ont eu les meilleures performances. Elles ont produit environ 03 feuilles après 40 jours en pépinière. La variété Safari a eu les plantules les plus grandes de taille avec environ 17 $\mathrm{cm}$ de hauteur. Ces plantules ont eu également les poids frais les plus élevés (423,11 mg). Les variétés Damani et Karibou ont eu les taux les plus élevés de matière sèche avec une valeur moyenne de $10,50 \%$. La variété Bati a eu le taux le plus faible de matière sèche $(08,14 \%)$.

En alvéole, à l'exception de la variété Damani, les autres variétés ont eu des hauteurs de plantules identiques d'environ $18 \mathrm{~cm}$. Les plantules de la variété Karibou ont produit plus de feuilles (03) que les autres. Les variétés Damani et Karibou ont eu les taux les plus élevés de matière sèche qui fluctuent entre 10,43 et $10,78 \%$.

Tableau 1. Caractéristiques morphologiques des plantules des cinq variétés d'oignon en pépinière sur terre

\begin{tabular}{|c|c|c|c|c|c|c|}
\hline Variétés & $\begin{array}{c}\text { Hauteur } \\
\text { plantule } \\
(\mathbf{c m})\end{array}$ & $\begin{array}{c}\text { Diamètre } \\
\text { plantule } \\
(\mathbf{c m})\end{array}$ & $\begin{array}{c}\text { Nombre } \\
\text { de feuille }\end{array}$ & $\begin{array}{c}\text { Poids } \\
\text { frais } \\
\text { (mg) }\end{array}$ & $\begin{array}{c}\text { Poids sec } \\
\text { (mg) }\end{array}$ & \% MS \\
\hline Bati & $14,29 \pm$ & $0,18 \pm 0,09$ & $2,30 \pm$ & $249,58 \pm$ & $20,67 \pm$ & $08,14 \pm$ \\
& $2,39 \mathrm{~b}$ & & $0,51 \mathrm{~b}$ & $62,33 \mathrm{~d}$ & $07,59 \mathrm{~d}$ & $1,57 \mathrm{c}$ \\
\hline Cara & $15,03 \pm$ & $0,20 \pm$ & $2,30 \pm$ & $309,39 \pm$ & $24,86 \pm$ & $09,49 \pm$ \\
& $3,71 \mathrm{~b}$ & 0,13 & $0,57 \mathrm{~b}$ & $156,53 \mathrm{c}$ & $08,47 \mathrm{c}$ & $3,72 \mathrm{~b}$ \\
\hline Damani & $15,36 \pm$ & $0,19 \pm$ & $\mathbf{2 , 4 9} \pm$ & $323,11 \pm$ & $33,97 \pm$ & $\mathbf{1 0 , 7 8} \pm$ \\
& $3,01 \mathrm{~b}$ & 0,07 & $\mathbf{0 , 5 3} \mathbf{~ a}$ & $119,62 \mathrm{c}$ & $11,52 \mathrm{~b}$ & $\mathbf{2 , 7 2} \mathbf{~ a}$ \\
\hline Karibou & $15,43 \pm$ & $0,20 \pm$ & $\mathbf{2 , 6 3} \pm$ & $366,87 \pm$ & $\mathbf{3 7 , 5 8} \pm \mathbf{0}$ & $\mathbf{1 0 , 4 3} \pm$ \\
& $2,64 \mathrm{~b}$ & 0,05 & $\mathbf{0 , 5 1} \mathbf{a}$ & $115,15 \mathrm{~b}$ & $\mathbf{9 , 6 8} \mathbf{a}$ & $\mathbf{0 , 9 7} \mathbf{a}$ \\
\hline Safari & $\mathbf{1 6 , 9 9} \pm$ & $0,20 \pm$ & $\mathbf{2 , 6 7} \pm$ & $\mathbf{4 2 3 , 1 1} \pm$ & $\mathbf{3 9 , 2 3} \pm$ & $09,44 \pm$ \\
& $\mathbf{3 , 5 4} \mathbf{a}$ & 0,04 & $\mathbf{0 , 5 4} \mathbf{a}$ & $\mathbf{1 2 1 , 0 7} \mathbf{a}$ & $\mathbf{0 9 , 7 3} \mathbf{a}$ & $1,24 \mathrm{~b}$ \\
\hline F & 9,17 & 1,30 & 9,85 & 26,88 & 65,92 & 18,22 \\
\hline P value & $<0,001$ & 0,27 & $<0,001$ & $<0,001$ & $<0,001$ & $<0,001$ \\
\hline
\end{tabular}

NB : En gras les valeurs les plus élevées. Sur une même colonne, les moyennes affectées de la même lettre sont statistiquement égales au seuil $\alpha=5 \%$ selon le test de Newman et Keuls. $\mathrm{F}=$ Statistique du test $; \mathrm{P}=$ probabilité

Source: Résultat projet de recherche sur l'oignon de l’UPGC (année 2020) 
Tableau 2. Caractéristiques morphologiques des plantules des cinq variétés d'oignon en pépinière dans les alvéoles

\begin{tabular}{|c|c|c|c|c|c|c|}
\hline Variétés & $\begin{array}{c}\text { Hauteu } \\
\mathbf{r} \\
\text { plantule } \\
(\mathbf{c m})\end{array}$ & $\begin{array}{c}\text { Diamètre } \\
\text { plantule } \\
\mathbf{( c m )}\end{array}$ & $\begin{array}{c}\text { Nombre } \\
\text { de feuille }\end{array}$ & $\begin{array}{c}\text { Poids } \\
\text { frais } \\
\mathbf{( m g})\end{array}$ & $\begin{array}{c}\text { Poids sec } \\
\mathbf{( m g )}\end{array}$ & \% MS \\
\hline Bati & $\mathbf{1 8 , 3 9} \pm$ & $0,20 \pm$ & $2,10 \pm$ & $\mathbf{3 4 4 , 6 0} \pm$ & $\mathbf{3 3 , 9 7} \pm$ & $9,86 \pm$ \\
& $\mathbf{1 , 4 8} \mathbf{a}$ & 0,01 & $0,32 \mathrm{~b}$ & $\mathbf{1 , 9 0} \mathbf{a}$ & $\mathbf{2 , 1 2} \mathbf{a}$ & $0,62 \mathrm{~b}$ \\
\hline Cara & $\mathbf{1 7 , 8 2} \pm$ & $0,18 \pm$ & $2,10 \pm$ & $331 \pm$ & $20,50 \pm$ & $6,19 \pm$ \\
& $\mathbf{2 , 2 8} \mathbf{a}$ & 0,04 & $0,32 \mathrm{~b}$ & $3,16 \mathrm{~b}$ & $1,58 \mathrm{~d}$ & $0,49 \mathrm{c}$ \\
\hline Damani & $15,05 \pm$ & $0,20 \pm$ & $1,90 \pm$ & $213,25 \pm$ & $24,60 \pm$ & $\mathbf{1 1 , 5 4} \pm$ \\
& $1,26 \mathrm{~b}$ & 0,01 & $0,32 \mathrm{~b}$ & $2,37 \mathrm{~d}$ & $1,90 \mathrm{c}$ & $\mathbf{0 , 9 1} \mathbf{~ a}$ \\
\hline Karibou & $\mathbf{1 7 , 9 1} \pm$ & $0,20 \pm$ & $\mathbf{2 , 5 0} \pm$ & $311 \pm$ & $20,40 \pm$ & $6,56 \pm$ \\
& $\mathbf{1 , 7 2} \mathbf{a}$ & 0,01 & $\mathbf{0 , 5 3} \mathbf{a}$ & $3,16 \mathrm{c}$ & $1,26 \mathrm{~d}$ & $0,42 \mathrm{c}$ \\
\hline Safari & $\mathbf{1 7 , 4 5} \pm$ & $0,18 \pm$ & $2,00 \pm 00$ & $305 \pm$ & $30,50 \pm$ & $10,02 \pm$ \\
& $\mathbf{1 , 2 1} \mathbf{a}$ & 0,03 & $\mathrm{~b}$ & $15,81 \mathrm{c}$ & $1,58 \mathrm{~b}$ & $0,73 \mathrm{~b}$ \\
\hline F & 6,45 & 2,00 & 4,50 & 475,29 & 125,38 & 125,92 \\
\hline Pvalue & $<0,001$ & 0,11 & $<0,001$ & $<0,001$ & $<0,001$ & $<0,001$ \\
\hline
\end{tabular}

NB : En gras les valeurs les plus élevées. Sur une même colonne, les moyennes affectées de la même lettre sont statistiquement égales au seuil $\alpha=5 \%$ selon le test de Newman et Keuls. $\mathrm{F}=$ Statistique du test $; \mathrm{P}=$ probabilité

Source: Résultat projet de recherche sur l'oignon de l’UPGC (année 2020)

Le tableau 3 présente le lien entre les variables mesurées. Le poids frais de la plantule est lié significativement à la hauteur $(\mathrm{r}=0,61)$, au diamètre $(\mathrm{r}=$ $0,54)$ et au nombre de feuilles $(r=0,72)$. Le poids sec est également corrélé significativement au diamètre $(r=0,55)$ et au nombre de feuilles $(r=0,68)$. Le Poids sec dépend du poids frais $(\mathrm{r}=0,84)$ et le taux de matière sèche croît avec le poids sec $(\mathrm{r}=0,53)$.

Tableau 3. Corrélation (r) de Pearson entre les variables mesurées chez l'oignon en pépinière

\begin{tabular}{|c|c|c|c|c|c|c|}
\hline Paramètres & $\begin{array}{l}\text { Hauteur } \\
\text { plantule }\end{array}$ & $\begin{array}{l}\text { Diamètre } \\
\text { plantule }\end{array}$ & $\begin{array}{l}\text { Nombre } \\
\text { de feuille }\end{array}$ & Poids frais & $\begin{array}{c}\text { Poids } \\
\text { sec }\end{array}$ & $\begin{array}{c}\% \\
\text { MS }\end{array}$ \\
\hline $\begin{array}{l}\text { Hauteur } \\
\text { plantule }\end{array}$ & 1 & 0,36 & 0,10 & $0,61^{* *}$ & 0,41 & $-0,21$ \\
\hline $\begin{array}{l}\text { Diamètre } \\
\text { plantule }\end{array}$ & & 1 & 0,38 & $0,54^{*}$ & $0,55^{*}$ & 0,40 \\
\hline $\begin{array}{c}\text { Nombre de } \\
\text { feuille }\end{array}$ & & & 1 & $0,72^{* *}$ & $0,68^{* *}$ & 0,11 \\
\hline Poids frais & & & & 1 & $0,84^{* *}$ & 0,05 \\
\hline Poids sec & & & & & 1 & $0,53^{*}$ \\
\hline$\% \mathrm{MS}$ & & & & & & 1 \\
\hline
\end{tabular}

Source: Résultat projet de recherche sur l'oignon de l’UPGC (2020) 


\section{Discussion}

Dans cette étude, les plantules élevées dans les alvéoles ont enregistré les plus grandes tailles. La croissance d'une plante dépend de plusieurs facteurs dont la richesse du milieu en éléments minéraux (Freschet et al., 2018). Ces minéraux sont partagés par les plantes cultivées sur une même superficie; par conséquent, le nombre ou la densité d'individus pourrait influencer le partage de ces minéraux. Dans cet essai, chaque poquet de l'alvéole contient trois plantules, chaque plantule a eu les minéraux nécessaires à sa croissance. À l'opposé, sur terre, la densité élevée de semis a influencé négativement la hauteur des plantules à telle enseigne que la dose de $05 \mathrm{~g} / \mathrm{m}^{2}$ a enregistré les plantules de taille relativement faible. Cela expliquerait aussi les faibles valeurs de diamètre au collet des plantules de la dose $05 \mathrm{~g} / \mathrm{m}^{2}$. Le substrat utilisé pour la conduite de la pépinière en alvéole apporterait les minéraux nécessaires à la croissance des plantules. Par ailleurs certains auteurs considèrent les plantules dont la hauteur est comprise entre 15 et $20 \mathrm{~cm}$ comme plus aptes pour le repiquage au champ (Abdou et al., 2015). Les plantules élevées en alvéole qui ont eu une forte vitesse de croissance avec des plantules relativement grandes de taille seraient plus aptes pour le repiquage au champ après 40 jours en pépinière.

La conduite de la pépinière dans les alvéoles a l'avantage de limiter les dégâts dus aux parasites du sol et adventices. En effet, l'oignon est une culture sensible à la compétition des adventices (Bennacer \& Bouderbala, 2016). Le coût relativement élevé de la conduite de la pépinière en alvéole pourrait limiter son utilisation par les paysans. La pépinière en pleine terre est la plus couramment rencontrée parce qu'elle demande peu d'investissement, cependant elle présente beaucoup de risques. En effet, elle est favorable aux fontes des semis, à l'attaque des parasites du sol, aux dégâts d'animaux et aux compétitions avec les adventices (Napo, 2013 ; Bello et al., 2019b).

Le nombre élevé de feuilles produites par les plantules ainsi que les valeurs élevées des poids frais, sec et taux de matière sèche des doses 03 et 04 $\mathrm{g} / \mathrm{m}^{2}$ militent en faveur de ces doses pour la conduite de la pépinière sur terre, cependant la dose de $04 \mathrm{~g} / \mathrm{m}^{2}$ pourrait avoir l'avantage du nombre élevé de plantules en pépinière.

La matière fraîche d'une plante dépendrait de la quantité d'eau dans la plante. Par ailleurs les différents organes de la plante contenant de l'eau ou les plantes qui ont plus de feuilles ont les poids frais les plus élevés et sont de grande taille. Cela explique les corrélations positives et significatives entre le poids frais et les variables "nombre de feuilles" et "hauteur de la plantule" révélées par les présents travaux.

Dans cette étude, au niveau de la pépinière conduite sur terre, les variétés Damani, Karibou et Safari ont eu les meilleures performances. Ces génotypes s'adapteraient mieux aux conditions de l'essai. Cependant lorsque 
la méthode de conduite change (en alvéole), les variétés Bati et Cara ont presque les mêmes performances que les autres variétés. De telles réponses laissent apparaître l'influence négative ou positive de la méthode de conduite diversement sur les génotypes d'oignon. Les variétés Damani, Karibou et Safari qui ont maintenu une relative performance quelle que soit la méthode de conduite de la pépinière, et pourraient mieux se comporter au champ après repiquage. En effet, la réussite du champ dépend en grande partie de la qualité des plants en pépinière (Assi et al., 2018).

Cette étude a révélé que le taux de matière sèche évolue positivement avec le poids sec et ce dernier dépend significativement du poids frais. Le poids de la plantule dépend significativement de sa hauteur et du nombre de feuilles. Les présents résultats indiquent que la sélection des plantules en pépinière peut se faire directement en se basant uniquement sur la taille des plantules et le nombre de feuilles. En d'autres termes, les plantules qui ont une bonne taille et qui produisent beaucoup de feuilles peuvent être considérées comme vigoureuses et doivent être sélectionnées pour le repiquage au champ.

\section{Conclusion}

Dans la présente étude, deux méthodes de conduite de la pépinière de l'oignon ont été évaluées à travers les caractéristiques morphologiques de cinq variétés que sont Bati, Karibou, Cara, Damani et Safari. À cet effet, les plantules soumises à trois doses de semis $\left(03,04\right.$ et $\left.05 \mathrm{~g} / \mathrm{m}^{2}\right)$ sur terre, et 03 graines par poquet alvéolée ont été mises en comparaison.

Il ressort de cette étude que les plantules élevées dans les alvéoles ont eu en moyenne les plus grandes tailles $(17,32 \mathrm{~cm})$. Sur terre, les tailles ont varié de $14,77 \mathrm{~cm}$ à $16,09 \mathrm{~cm}$ respectivement pour les doses de $05 \mathrm{~g} / \mathrm{m}^{2}$ et 03 et $04 \mathrm{~g} / \mathrm{m}^{2}$. Les doses de semis 03 et $04 \mathrm{~g} / \mathrm{m}^{2}$ ont enregistré les plantules les plus robustes relativement au nombre de feuilles (03) et les poids frais et sec. Les variétés Damani, Karibou et Safari ont eu les meilleures performances relativement aux caractéristiques morphologiques. La robustesse de la plantule est corrélée positivement à sa taille et au nombre de feuilles. Au vu des résultats, la pépinière d'oignon peut être conduite dans les alvéoles et la dose de $04 \mathrm{~g} / \mathrm{m}^{2}$ peut être conseillée pour la conduite de la pépinière sur terre. La sélection des plantules en pépinière peut se faire directement en se basant uniquement sur la taille de la plantule et le nombre de feuilles.

\section{Remerciements}

Les auteurs remercient le KAFACI (Korea Africa Food \& Agriculture Cooperation Initiative) pour le financement de cette étude. 


\section{References:}

1. Assi E.M., Dogbo O.D., Kassin E., Assiri A.A., Tahi G.M., Guiraud B., N’guessan W.P., Aka R.A., N’guessan F. \& Kone B. (2018). Détermination de l'âge optimal en pépinière des plants de cacaoyer pour une meilleure réussite au champ. African Crop Science Journal 26(4): 491 - 501.

2. Abdou R., Malice M., Bakasso Y., Saadou M. \& Baudoin J.P. (2015). Variabilité morphologique et agronomique des écotypes d'oignon (Allium cepa L.) identifiés par les producteurs du Niger. TROPICULTURA 33 (1) : 3-18.

3. Bello S., Ahanchede A. \& Amadji G.L. (2019a). Influence des pratiques de désherbage manuel sur l’évolution à long terme de l'enherbement et du rendement de l'oignon (Allium cepa L) au NordEst du Bénin, Afrique SCIENCE 15(6) : $11-26$.

4. Bello S.Ahanchede A. \& Amadji G. L. (2019b). Détermination des périodes de compétition des mauvaises herbes en culture d'oignon (Allium cepa L.) au Nord-Est du Bénin. Int. J. Biol. Chem. Sci. 13(6): 2497-2512

5. Bennacer M. \& Bouderbala A. (2016). Etude du désherbage (chimique et manuel) en pépinière sur la culture d'oignon A. cepa . (Deux hybrides F1 et une variété population). Mémoire de Master en AGRONOMIE, Université Abdelhamid Ibn Badis-Mostaganem, Algérie, 73 p.

6. David-Benz H. \& Seck A. (2018). Améliorer la qualité de l’oignon au Sénégal: contractualisation et autres mesures transversales. Rapport d'analyse de politique, SAPAA (projet de Suivi et Analyse des Politiques Agricoles et Alimentaires). Rome, FAO, 50 P.

7. Freschet G.T., Violle C., Roumet, C. \& Garnier E. (2018). Interactions entre le sol et la végétation : structure des communautés de plantes et fonctionnement du sol. Les sols au cœur de la zone critique : écologie (eds P. Lemanceau \& M. Blouin), pp. 83-99. ISTE éditions, London, UK.

8. Kiema N. (2019). Warrantage de l'oignon et autonomisation financière de la femme en milieu rural, défis et perspectives : cas du groupement féminin maraîcher relwindé de boulounsi dans le sous bassin versant de zogoré. Mémoire de fin de cycle Diplome d’Ingenieur du Developpement Rural. Université Nazi Boni (UNB), Institut du Developpement Rural (IDR), Burkina Faso, 51 p.

9. Laouali A., Assoumane A., Abdou M. M., Abdoulkadri A. \& Alzouma M.Z. (2019). Analyse de la Chaine de Valeurs d’oignon (Allium Cepa L.) Blanc de Soucoucoutane au Niger. European Scientific Journal 15, (3): 99-117. 
10. Napo H. I. (2013). Etude diagnostique des techniques de production de l'oignon (Allium cepa L.) dans la province du Yatenga. Memoire de fin de cycle en vue de l'obtention du Diplôme d'ingénieur en vulgarisation agricole, Université polytechnique de Bobo-Dioulasso (Burkina Faso), 53 p.

11. RONGEAD (2014). Diagnostic de la filière oignon en côte d'ivoire. Projet « Redynamiser les productions, l'accès au marché et le conseil agricole pour les filières vivrières et commerciales du Nord de la Côte d’Ivoire ». Coopération Union Européenne - République de Côte d'Ivoire Programme FLEX-FED/2013/324 233, 35p.

12. Siéné L. A.C., Doumbouya M., Traoré M.S., Condé M., N’guettia T.V.F. \& Koné M. (2020). Effet de quatre types de fertilisants sur la croissance et la productivité de deux génotypes de maïs (Zea mays L.) en cas d'un semis tardif à Korhogo au Centre-Nord de la Côte d'Ivoire, Int. J. Biol. Chem. Sci. 14(1): 55-68.

13. Silué S., Fondio L., Coulibaly M.Y. \& Magein H. (2003). Sélection de variétés d'oignon (Allium cepa L.) adaptées au nord de la Côte d'Ivoire, TROPICULTURA (21), 3, 129-134. 